\title{
Factores de riesgo asociados a reacción adversa hepática por fármacos de primera línea contra Mycobacterium tuberculosis
}

\author{
FERNANDO TIRAPEGUI S.* y CARLOS PEÑA M.**
}

\section{Risk factors associated with adverse hepatic reaction by first-line drugs against Mycobacterium tuberculosis}

Epidemiological control of tuberculosis (TB) requires a highly efficient therapy, to be able to eliminate transmission of tuberculosis. Adverse drug reactions (ADR) contribute to decrease the efficiency of treatment. Hepatic ADR is the most feared and can become fatal and has been related to risk factors such as advanced age, female gender, non-caucasian ethnic groups, characteristics of enzymatic metabolism of drugs, drug associations, presence of hepatitis B, C and HIV virus infections, malnutrition, kidney transplants, pregnancy, puerperium and alcohol consumption. There are no guidelines for monitoring liver function on a regular basis. Therefore a close monitoring of the appearance of symptoms suggestive of liver toxicity is recommended. In some conditions of high risk of hepatic ADR, clinical judgment could indicate the biochemical monitoring of liver function.

Key words: Drug related side effects and adverse reactions; Risk factors; Tuberculosis.

\section{Resumen}

Para el control epidemiológico de la tuberculosis (TBC) se requiere de una terapia de alta eficacia, que logre eliminar la trasmisión mediante la curación de los pacientes. Las reacciones adversas a fármacos (RAM) contribuyen a una menor eficiencia del tratamiento. La RAM hepática es la más temida y puede llegar a ser mortal. Esta se ha relacionado a factores de riesgo como la edad avanzada, el género femenino, ciertas razas, características del metabolismo enzimático de los fármacos, asociación de fármacos, presencia de infecciones por virus de hepatitis B, C y VIH, desnutrición, trasplantados renales, embarazo, puerperio y consumo de alcohol. No existen pautas de monitoreo de la función hepática en forma regular, por lo que se recomienda la vigilancia estrecha de la aparición de síntomas sugerentes de toxicidad hepática. En algunas condiciones de alto riesgo de RAM hepática el juicio clínico podría determinar el monitoreo bioquímico de la función hepática.

Palabras clave: Efectos secundarios y reacciones adversas por drogas; Factores de riesgo; Tuberculosis.

\section{Introducción}

Para reducir la transmisión de la tuberculosis (TBC) es necesario mejorar la cobertura y oportunidad de pesquisa y terapia de los casos de TBC pulmonar bacteriológicamente demostrada, especialmente de los pacientes bacilíferos. Un factor que influye en la eficiencia del tratamiento son las reacciones adversas a los medicamentos (RAM) utilizados, lo que determina la necesidad de emplear esquemas más prolongados, con menor adherencia, riesgo de abandono, menor seguridad (con más toxicidades) y menos eficaces (con menor poder bactericida y esterilizante).

* Departamento Enfermedades Respiratorias, Facultad de Medicina, Pontificia Universidad Católica de Chile. Santiago, Chile.

** Encargado de Programa de Control y Eliminación de la Tuberculosis (PROCET), Servicio de Salud Metropolitano Central. Santiago, Chile. 
Además, estas RAM pueden requerir de hospitalizaciones, incrementar los costos sanitarios y hay algunas además lo suficientemente severas como para ocasionar la muerte. Las reacciones más comunes asociadas con los medicamentos de primera línea (isoniazida $=\mathrm{H}$, rifampicina $=$ $\mathrm{R}$, etambutol $=\mathrm{E}$ y Pirazinamida $=\mathrm{Z}$ ) son de naturaleza gastrointestinal y cutánea ${ }^{1,2}$. Entre el $25-60 \%$ de los pacientes informan al menos algún tipo de $\mathrm{RAM}^{3-5}$. Una RAM de gran importancia clínica es la hepatotoxicidad, la cual se ha asociado con tres medicamentos de primera línea: $\mathrm{H}, \mathrm{R}$ y $\mathrm{Z}^{1,6-13}$. De los tres fármacos, la $\mathrm{R}$ tiene menos probabilidades de causar daño hepatocelular ${ }^{14}$, aunque puede asociarse con ictericia colestática $^{11,12}$. La $Z$ es la más hepatotóxica de los medicamentos de primera línea ${ }^{1,11,12}$. La mayoría de las hepatitis inducidas por medicamentos se produce dentro de los primeros 2 meses de tratamiento ${ }^{7,10}$ y raramente produce muertes por necrosis hepática fulminante ${ }^{15-18}$. El espectro de hepatotoxicidad por fármacos anti $\mathrm{TBC}$ va desde una elevación transitoria de las transaminasas en un $25 \%$ de los pacientes, causada por inducción enzimática que puede aparecer en los 2 primeros meses de terapia, hasta generar el daño hepático inducido por droga o Drug Induced Liver Injury (DILI). DILI representa el 7\% de los efectos adversos informados de los medicamentos, el $2 \%$ de las ictericias en los hospitales y aproximadamente el 30\% de los casos de insuficiencia hepática fulminante ${ }^{19}$. Por lo descrito respecto a la toxicidad hepática y sus implicancias, resulta muy relevante para el clínico conocer e identificar los factores asociados a una mayor probabilidad de esta RAM, lo que motiva este trabajo de evaluación de estos factores en las fuentes de información científica.

\section{Materiales y Método}

Se realizó una búsqueda en PubMed utilizando los términos "factor de riesgo", "tuberculosis", "hepatotoxicidad" y "medicamentos antituberculosos" para identificar artículos adecuados para la literatura de referencia. Se consideraron artículos originales, reseñas, editoriales y sus listas de referencias. No hubo restricciones de idioma. Generamos una pregunta relacionada al factor de riesgo con la metodología PICO (traducción de P: Patient/Problem; I: Intervention; C: Comparison y O: Outcome), mediante la cual se define una condición o problema de un paciente o de una población y se desea conocer si hay evidencias de como se ha enfrentado ese problema y los resul- tados comparativos de las diferentes estrategias de intervención.

\section{Resultados}

\section{Pregunta 1. ¿Es la edad un factor de riesgo para la hepatotoxicidad por fármacos antitu- berculosos de primera línea?}

El principal estudio fue realizado por Kopanoff para el CDC el año $1978^{20}$, en 13.838 sujetos sometidos a terapia con $\mathrm{H}$ por al menos un mes se encontró 174 casos con hepatitis. El riesgo de hepatitis es mayor sobre los 35 años (entre 35 a 49 años: 12 casos por cada 1.000 personas; entre 50 a 64 años: 23 casos por 1.000 personas y en mayores de 64 años: 8 casos por cada 1.000 personas). Van den Brande el año 1995 realizó un estudio ${ }^{21}$ en pacientes sometidos a esquema con $\mathrm{H}$ y $\mathrm{R}$, con ausencia de patología hepática basal y en el cual definió como punto de corte los 60 años en búsqueda de RAM. Los aumentos de transaminasas por encima de los valores iniciales fueron más frecuentes en mayores de 60 años (38 versus $18 \%, p<0,05$ ). En un estudio ${ }^{22}$ con esquema asociado a $Z$, la hepatotoxicidad severa se produjo con mayor frecuencia entre los 15 y 25 años con dosis de $Z$ ajustada al peso $\geq 30 \mathrm{mg} / \mathrm{kg}$.

\section{Pregunta 2. ¿Es el género un factor de riesgo?}

No hay estudios concluyentes de una predilección hacia un sexo definido, pero se observa una tendencia en un gran número de estudios a un desenlace desfavorable y mayor compromiso hepático en mujeres. En un estudio de 1978 más de la mitad de las reacciones leves eran en mujeres. La misma tendencia prevaleció en pacientes que mostraron un gran aumento de transaminasas, pero la diferencia con el grupo control, no fue significativa ${ }^{23}$. Yee y Valiquette ${ }^{1}$ encontraron que el sexo femenino se asoció significativamente con cualquier efecto secundario a drogas anti TBC. Las mujeres de edad avanzada constituyen un grupo de riesgo especial, con mayores reacciones tóxicas y alérgicas ${ }^{24}$. Iwainsky y cols informaron concentraciones sanguíneas más altas de $\mathrm{R}$ entre las mujeres ${ }^{25}$.

\section{Pregunta 3. ¿Es el embarazo un factor de riesgo?}

A petición de los NIH (National Institutes of Health, USA), los autores investigaron la morbilidad y mortalidad asociada a hepatitis por $\mathrm{H}$ entre las pacientes que asisten a control prenatal de origen hispano. Entre 3.681 mujeres tratadas con $\mathrm{H}$ durante y después del embarazo para prevenir la 
TBC, 5 desarrollaron DILI y de estas 2 fallecieron. Utilizando el modelo de riesgos proporcionales de Cox, reveló un riesgo 2,5 veces mayor de DILI en el grupo de embarazadas. La tasa de mortalidad fue cuatro veces mayor en el grupo de embarazadas. Sin embargo, el poder estadístico fue bajo debido al pequeño número de casos, y ninguno de estos hallazgos fue estadísticamente significativo $(p>0,05)^{26}$.

\section{Pregunta 4. ¿Es la raza un factor de riesgo?}

Gordin y cols. en un estudio poblacional para destacar que el uso de $\mathrm{R}$ y $\mathrm{Z}$ es factor de riesgo para presentar DILI en la raza no caucásica, lograron demostrar un aumento en el nivel de bilirrubina mayor de $0,5 \mathrm{mg} / \mathrm{dL}$ desde el inicio de la terapia hasta el mes 1 y 2 del estudio (OR: 2,26; $1,13-4,53$ IC $95 \%$ ) y un aumento de $40 \mathrm{U} / \mathrm{L}$ de AST (OR 1,43; 0,82-2,50 IC 95\%) ${ }^{27}$. Un segundo estudio realizado por Yee y cols. determinó que al comparar la raza "asiática" versus todas las otras razas, esta presenta un mayor riesgo de DILI (HR $2,2 ; 0,7-6,9)^{1}$.

\section{Pregunta 5. ¿Es la velocidad de acetilación un factor de riesgo para hepatotoxicidad?}

La N-acetiltransferasa 2 (NAT2) es la principal responsable del metabolismo de la $\mathrm{H}$. La tasa de eliminación de $\mathrm{H}$ se distribuye trimodalmente de acuerdo con el fenotipo/genotipo de NAT2. El polimorfismo genético puede ser de gran importancia para modificar los efectos tóxicos de la terapia con medicamentos antituberculosos ${ }^{28}$. En estudios de las décadas de los 70's y 80's se caracterizaba a los acetiladores rápidos como un factor de riesgo para DILI con una dudosa calidad metodológica. Los estudios realizados en la segunda mitad de los años 80's empiezan a mostrar evidencia de mejor calidad apoyada con hallazgos fisiopatológicos logran determinar que la acetilación rápida es un factor protector y que en cambio los acetiladores lentos al mantener niveles plasmáticos elevados de fármacos anti TBC son un factor de riesgo para presentar hepatotoxicidad por fármacos anti $\mathrm{TBC}^{29}$. Huang y cols, genotipificaron NAT2 en 224 pacientes con TBC que recibieron tratamiento antituberculoso $^{30}$. En 33 pacientes $(14,7 \%)$ con hepatitis inducida por medicamentos antituberculosos, los acetiladores lentos tenían mayor riesgo de hepatotoxicidad que los acetiladores rápidos $(26,4 \%$ versus $11,1 \%, p=0,013)$. En los pacientes con hepatotoxicidad, los acetiladores lentos tenían niveles de aminotransferasas séricas significativamente más elevados que los acetiladores rápidos. La regresión logística mostró que el estado de acetilador lento (OR: 3,66; IC 95\%, 1,58-8,49; $\mathrm{p}=0,003)$ y la edad (OR 1,09 ; IC $95 \%, 1,04-$ $1,14 ; \mathrm{p}<0,001)$ fueron los únicos dos factores de riesgo independientes para la hepatitis inducida por fármacos antituberculosos.En conclusión, el estado de acetilador lento de NAT2 es un factor de riesgo de susceptibilidad significativo para la hepatitis inducida por fármacos antituberculosos. Además, son propensos a desarrollar una hepatotoxicidad más severa que los acetiladores rápidos. Ohno encontró que la incidencia de elevación de la aminotransferasa sérica fue baja en los acetiladores rápidos $(3,5 \%)$ y alta en los acetiladores lentos $(100 \%)^{28}$. Diversos estudios han propuesto la hipótesis de que la $\mathrm{H}$ compite con acetilhidrazina por la vía de acetilación, y que existiría más acetilhidrazina disponible en el metabolismo oxidativo para formar radicales activos en aquellos sujetos acetiladores lentos con el consiguiente riesgo de hepatotoxicidad ${ }^{31-35}$.

\section{Pregunta 6. ¿Existen otras variaciones genéticas de riesgo?}

Otras enzimas posibles son CYP2E1 y glutatión S-transferasa. Existe evidencia de que los polimorfismos de los genes que codifican estas enzimas pueden influir en la actividad de las enzimas metabolizadoras de fármacos. Estudios recientes demuestran que los polimorfismos genéticos pueden estar asociados con la susceptibilidad a la lesión hepática inducida por fármacos antituberculosos. Los genotipos asociados al riesgo propuestos son el acetilador lento NAT2 ( $\sin$ el alelo natural NAT2 $* 4$ ), CYP2E1 * 1A / * $1 \mathrm{~A}$ (homocigótico de tipo salvaje) y el genotipo nulo homocigótico GSTM1. Los datos disponibles son aún limitados y se requiere estudios en diferentes poblaciones étnicas con tamaños de muestra más grandes. Resulta interesante la aplicación de enfoques fármaco-genómicos para prevenir graves lesiones hepáticas inducidas por fármacos antituberculosos en el futuro cercano ${ }^{36}$.

\section{Pregunta 7. ¿Es el alcohol un factor de riesgo?}

Lönnroth y cols. mostraron que el consumo de alcohol de más de $40 \mathrm{~g}$ de etanol por día o el diagnóstico de un trastorno por consumo de alcohol se asoció con un riesgo casi tres veces mayor de tuberculosis (RR: 2,94; IC 95\%: 1,894,59 ) en comparación con ausencia de consumo de alcohol o el consumo de alcohol por debajo de los umbrales definidos ${ }^{37}$. En datos publicados en los años 70's ${ }^{23}$, la ingesta alta de alcohol definido como una ingesta semanal media en el último año de $280 \mathrm{~g}$ de etanol para hombres y $140 \mathrm{~g}$ de etanol para mujeres fue un factor de 
riesgo para el desarrollo de la hepatitis inducida por los fármacos ${ }^{38}$. En una serie de estudios entre quienes presentan un alto consumo de alcohol, se registró la presencia de hepatotoxicidad entre el 8 a 19\% de los pacientes, lo que indica que los consumidores de alcohol son más propensos a desarrollar toxicidad ${ }^{29,39-42}$. En un reciente estudio pakistaní el consumo de alcohol demostró ser un factor de riesgo de hepatotoxicidad $(25,5 \%)$ en comparación a quienes no consumían alcohol ${ }^{43}$. En el estudio de vigilancia del Servicio de Salud Pública de Estados Unidos (USPHS), el consumo de alcohol duplica la tasa de hepatitis probable por $\mathrm{H}$, y el consumo diario aumenta la tasa más de cuatro veces ${ }^{39}$. En un estudio de simulación realizado en Brasil, Uganda y la India, en donde a sujetos portadores de HIV y con alto consumo de alcohol se les administró terapia preventiva con $\mathrm{H}$ (IPT) y terapia antirretroviral (ART) por 6 (IPT6) y 36 (IPT36) meses en comparación a ART sola. La toxicidad se produjo en $160 / 1.000$ personas en IPT6, y 415/1.000 personas en IPT36, con toxicidad fatal en 8/1.000 en IPT6 y 21/1.000 en IPT36 $6^{44}$.

\section{Pregunta 8. ¿La extensión de la tuberculosis es un factor de riesgo?}

La extensión de la enfermedad también fue un factor de riesgo importante para el desarrollo de la hepatitis y ha sido definida como la presencia de baciloscopia positiva, TBC cavitada o TBC con compromiso abdominal ${ }^{29,43}$.

\section{Pregunta 9. ¿La desnutrición puede ser un factor de riesgo para hepatotoxicidad?}

Los pacientes con hipoalbuminemia previa al tratamiento tienen un riesgo dos veces mayor de desarrollar hepatotoxicidad. En la desnutrición, las reservas de glutatión se agotan, lo que hace al organismo vulnerable a la lesión oxidativa. En una persona desnutrida, el hígado metaboliza las drogas a un ritmo más lento. En un estudio realizado en India, se encontró que la incidencia de hepatotoxicidad era 3 veces mayor en pacientes desnutridos ${ }^{45,46}$.

\section{Pregunta 10. ¿La infección con virus de hepatitis $B, C y$ VIH son factores de riesgo para hepatotoxicidad por fármacos antituberculosos?}

Wong y cols. estudiaron la disfunción hepática durante el tratamiento antituberculoso en portadores del virus de la hepatitis B (VHB) y no portadores ${ }^{47}$. Cuarenta y tres pacientes con VHB y 276 pacientes sin VHB se incluyeron en el análisis. La incidencia de disfunción hepática fue significativamente mayor en los portadores de VHB que recibieron fármacos antituberculosos $(34,9 \%)$ en comparación con los no portadores $(9,4 \%, p<0,001)$ y con los portadores de VHB que no recibieron fármacos antituberculosos $(8,1 \%, \mathrm{p}<0,001)$. Hoffmann y cols. ${ }^{48}$ en un estudio con 868 sujetos en África, 25\% recibió tratamiento concomitante de tuberculosis durante ART y $17 \%$ de un subconjunto seleccionado al azar fue positivo para antígeno de superficie de hepatitis B (HBsAg). Se registró 7,7 episodios de hepatotoxicidad severa por cada 100 personasaños. El tratamiento de tuberculosis aumentó el riesgo 8,5 veces, el $\mathrm{HBs} A g$ positivo 3,0 veces y el nadir de células CD4 $<100 / \mu \mathrm{L} 1,9$ veces. Un paciente que desarrolló hepatotoxicidad fue positivo tanto para HBSAg como para anti-VHC. Todos los pacientes con anticuerpos anti-VHC que desarrollaron hepatotoxicidad fueron positivos al ARN del VHC mediante la prueba de PCR cualitativa. En un estudio ${ }^{49}$ de 1998 se encontró que 12 de los 22 pacientes (55\%) que desarrollaron hepatotoxicidad resultaron positivos al VIH mediante ELISA y los resultados se confirmaron mediante el ensayo de Western blot. Un total de $5(4 \%)$ de los 128 pacientes en el estudio fueron positivos para HBSAg. De éstos, solo uno (20\%) desarrolló hepatotoxicidad. La presencia de VHC o VIH fue un factor de riesgo independiente significativo para el desarrollo de hepatotoxicidad. Los pacientes con infección por VIH reciben múltiples medicamentos para la infección primaria por VIH y para infecciones oportunistas, como la tuberculosis, infección por Pneumocystis jirovecci y hepatitis $\mathrm{B}$ y $\mathrm{C}^{50}$. La superposición de toxicidades e interacciones entre los fármacos, la enfermedad misma y la susceptibilidad del huésped pueden representar mayor riesgo de hepatotoxicidad. Los fármacos asociados con mayor riesgo de DILI incluyen zidovudina, estavudina, nevirapina, efavirenz, abacavir y otros ${ }^{50,51}$.

En infectados con hepatitis B y C crónica, es difícil determinar si el aumento de las transaminasas o la bilirrubina es inducido por fármacos $\mathrm{o}$ por un brote de hepatitis B o C.

\section{Pregunta 11. ¿Los receptores de trasplante renal tienen un mayor riesgo?}

La TBC es más frecuente en los receptores de trasplante renal que en la población general. En Turquía un estudio diagnosticó TBC en 22 de 520 receptores de trasplante renal con una prevalencia de $4,2 \%{ }^{52}$. Los pacientes recibieron protocolos de terapia triple (11 pacientes) o uno de los dobles (ciclosporina CycA + Prednisona PDN, 8 pacientes), (azatioprina AZA + PDN, 
3 pacientes). El intervalo de tiempo entre el trasplante y el diagnóstico de TBC fue de 44,4 $\pm 33,5$ meses (rango 3-111). En 18 (82\%) de los pacientes, la TBC se detectó después del primer año del trasplante. La creatinina sérica media fue de $2,0 \pm 1,6 \mathrm{mg} / \mathrm{dL}$ (rango 0,9 y 8,0 ) y solo en 3 pacientes la creatinina sérica fue $>2 \mathrm{mg} /$ $\mathrm{dL}$, en el momento del diagnóstico. Se describió que 4 pacientes tenían antecedentes de rechazo agudo y habían tomado altas dosis de esteroides para revertir el rechazo. El sitio más común fue pulmón ( 8 pacientes, $36 \%$ ) o pleura (4 pacientes, $18 \%$ ); es decir, la afectación pleuro-pulmonar representó más de la mitad de los casos. La TBC extrapulmonar aislada se diagnosticó en 7 (31\%) de los pacientes. Se observó hepatotoxicidad en $4(18 \%)$ pacientes. Después de la retirada de la $\mathrm{H}$, los niveles séricos de transaminasas volvieron rápidamente a la normalidad en la primera semana, excepto en un paciente que progresó a hepatitis crónica activa e insuficiencia hepática. La $\mathrm{H}$ se readministró en todos los casos sin observarse anormalidad en la transaminasa sérica. En otro estudio de trasplantados renales ${ }^{53}$ se encontró que, en el seguimiento, el $31 \%$ de los pacientes tenían una actividad de alanina amino transferasa (ALAT) anormal ( $>40$ UI), sin embargo, las elevaciones eran pequeñas (el nivel de ALAT más alto fue de $88 \mathrm{UI}$ ) y nunca necesitaron la suspensión de $\mathrm{H}$. Ningún paciente tuvo ictericia u otra evidencia de hepatotoxicidad clínica. El intervalo de confianza del 95\% para la frecuencia observada de hepatitis clínica fue del $0 \%$ al 4,3\%. Al final del tratamiento con $\mathrm{H}$, la ALAT promedio fue de $22,7 \pm 6,2 \mathrm{UI}$ ( $\mathrm{p}>$ 0,2 , en comparación con el inicio ALAT) y solo un paciente tenía un ALAT anormal.

En conclusión, parece que el riesgo de receptores de trasplante renal que desarrollan hepatotoxicidad grave con la administración de $\mathrm{H}$ es bajo.

\section{Pregunta 12. ¿La interacción farmacológica confiere un mayor riesgo?}

La interacción entre fármacos administrados de forma concomitante es compleja y desafiante y complica la evaluación de la causalidad. A menudo, los medicamentos pueden tener interacción recíproca de tal manera que cualquiera de los dos fármacos aumenta el potencial de hepatotoxicidad del otro. Por ejemplo, la carbamazepina y la $\mathrm{H}$ causan la inhibición del metabolismo de cualquiera de los fármacos, aumentando así los niveles sanguíneos de cada uno de los medicamentos $^{54}$. La Z, H y R son hepatotóxicas en orden de propensión decreciente ${ }^{55}$. La combinación de H y R es más hepatotóxica que cualquiera de los fármacos por sí solo. Esto se debe principalmente a que la $\mathrm{R}$ aumenta el potencial hepatotóxico de la H. Además, existe una inhibición relativa de la toxicidad de la $\mathrm{Z}$ al utilizar la $\mathrm{H}^{56}$, mientras que se apreciaron altas tasas de toxicidad hepática, incluso mortales, en pacientes tratados con la combinación de $\mathrm{R}$ y $\mathrm{Z}$ en algunas terapias de tuberculosis latente ${ }^{57}$.

\section{Discusión}

Aunque, existe cierta controversia con respecto a si debe realizarse la evaluación de la química hepática de rutina en el tratamiento de la $\mathrm{TBC}$, los pacientes con factores de riesgo de hepatotoxicidad deben someterse a un control bioquímico regular ${ }^{58,59}$. Los inconvenientes de la monitorización mediante seguimiento de sintomatología son su falta de especificidad y la demora en el diagnóstico debido al umbral sintomático elevado, especialmente en ancianos, alcohólicos o pacientes con enfermedades psiquiátricas ${ }^{11}$. Un pequeño estudio no aleatorizado ha sugerido que el control de la bioquímica hepática puede ayudar a prevenir la hepatotoxicidad grave ${ }^{60}$. Un reciente estudio de cohorte prospectivo mostró que un enfoque de monitoreo basado en el riesgo, como lo recomienda la ATS, evitó el 33,3\% de la hepatotoxicidad temprana y el $77,8 \%$ de la hepatotoxicidad tardía ocurrida dentro y después de 2 semanas de tratamiento, respectivamente ${ }^{61}$. La alteración de ALT (alanina transaminasa) después de 2 semanas de tratamiento tuvo una sensibilidad baja del $22,2 \%$ para la hepatotoxicidad posterior, independientemente de la presencia o ausencia de factores de riesgo al inicio del estudio. Por lo tanto, se requieren más estudios para delinear el papel exacto del monitoreo bioquímico regular en el tratamiento de la TBC. La ALT es más específica que la AST (aspartato amino transferasa) para la lesión hepatocelular ${ }^{62}$, ya que las elevaciones de esta última también pueden indicar anomalías en el músculo, el corazón o el riñón. Sin embargo, los cambios transitorios en los niveles de bilirrubina y transaminasas son relativamente comunes durante la terapia antituberculosa. Hasta el $15 \%$ de los pacientes tratados con isoniazida desarrollan elevaciones de ALT incluso superiores a tres veces el LSN, pero la gran mayoría de estos son transitorios y asintomáticos $^{63}$. A pesar del hecho de que los umbrales de ALT definidos por la ATS son limitantes del tratamiento actual, estos pueden no diferenciar con precisión entre la adaptación hepática y el inicio de una lesión hepática significativa. Los 
aumentos de ALT en combinación con las elevaciones del nivel de bilirrubina total son predictores específicos bien aceptados de lesión hepática grave inducida por fármacos ${ }^{64}$.

En un ensayo controlado aleatorizado o RCT (randomized controlled trial) ${ }^{65}$, el riesgo de hepatotoxicidad recurrente debido a la reintroducción simultánea de $\mathrm{H}, \mathrm{R}$ y $\mathrm{Z}(13,8 \%)$ no fue significativamente diferente de la reintroducción secuencial en dosis completas $(10,2 \%)$ o dosis gradualmente aumentadas $(8,6 \%)$. El riesgo del $13,8 \%$ de hepatotoxicidad recurrente no es bajo, teniendo en cuenta su curso altamente impredecible. Reintroducir un medicamento a la vez puede ser el enfoque óptimo, especialmente si la hepatitis del paciente es grave. Aún no se ha demostrado convincentemente que ningún agente hépato-protector sea útil durante la fase hepatotóxica.

\section{Conclusión}

El nivel de evidencia actual para definir factores de riesgo y pronóstico es de discreta calidad, lo que no permite realizar recomendaciones con un respaldo fuerte y sólido. Sin embargo, pensamos que se deben divulgar los factores relacionados al riesgo de hepatotoxicidad por drogas antituberculosas conocidos hasta la fecha, para que, en base al juicio clínico y acorde a la realidad local, se defina un seguimiento clínico más exhaustivo en este grupo de pacientes.

\section{Bibliografía}

1.- YEE D, VALIQUETTE C, PELLETIER M, PARISIEN I, ROCHER I, MENZIES D. Incidence of serious side effects from first-line antituberculosis drugs among patients treated for active tuberculosis. Am J Respir Crit Care Med 2003; 167: 1472-7.

2.- MARRA F, MARRA CA, BRUCHET N, RICHARDSON K, MOADEBI S, ELWOOD RK, et al. Adverse drug reactions associated with first-line anti-tuberculosis drug regimens. Int J Tuberc Lung Dis 2007; 11: 868-75.

3.- BRITISH THORACIC SOCIETY. A controlled trial of 6-months chemotherapy in pulmonary tuberculosis. Final report: Results during the 36-months after the end of chemotherapy and beyond. Br J Dis Chest 1984; 78: 330-6.

4.- HONG KONG CHEST SERVICE, BRITISH MEDICAL RESEARCH COUNCIL. Five-year follow-up of a controlled trial of five-month regimens of chemotherapy for pulmonary tuberculosis. Am Rev Respir Dis 1987; 136: $1339-42$.
5.- HONG KONG CHEST SERVICE, TUBERCULOSIS RESEARCH CENTRE MADRAS, BRITISH MEDICAL RESEARCH COUNCIL. A controlled trial of 3-month, 4-month and 6-month regimens of chemotherapy for sputum-smear-negative pulmonary tuberculosis. Results at 5 years. Am Rev Respir Dis 1989; 139: 871-6.

6.- MARRA F, MARRA CA, BRUCHET N, RICHARDSON K, MOADEBI S, ELWOOD RK, et al. Adverse drug reactions associated with first-line anti-tuberculosis drug regimens. Int J Tuberc Lung Dis 2007; 11: 868-75.

7.- GIRLING DJ. Adverse effects of antituberculosis drugs. Drugs 1982; 23: 56-74.

8.- SCHABERG T, REBHAN K, LODE H. Risk factors for side-effects of isoniazid, rifampin and pyrazinamide in patients hospitalized for pulmonary tuberculosis. Eur Respir J 1996; 9: 2026-30.

9.- DØSSING M, WILCKE JT, ASKGAARD DS, NYBO B. Liver injury during antituberculosis treatment: an 11-year study. Tuber Lung Dis 1996; 77: 335-40.

10.- SHAKYA R, RAO BS, SHRESTHA B. Incidence of hepatotoxicity due to antitubercular medicines and assessment of risk factors. Ann Pharmacother 2004; 38: 1074-79.

11.- YEW WW, LEUNG CC. Antituberculosis drugs and hepatotoxicity. Respirology 2006; 11: 699-707.

12.- SAUKKONEN JJ, COHN DL, JASMER RM, SCHENKER S, JEREB JA, NOLAN CM, et al. An official ATS statement: hepatotoxicity of antituberculosis therapy. Am J Respir Crit Care Med 2006; 174: 935-52.

13.- TOSTMANN A, BOEREE MJ, AARNOUTSE RE, DE LANGE WC, VAN DER VEN AJ, DEKHUIJZEN R. Antituberculosis drug-induced hepatotoxicity: concise up-to-date review. J Gastroenterol Hepatol 2008; 23: 192-202.

14.- HONG KONG CHEST SERVICE, TUBERCULOSIS RESEARCH CENTRE MADRAS, BRITISH MEDICAL RESEARCH COUNCIL. A double-blind placebo-controlled clinical trial of three antituberculosis chemoprophylaxis regimens in patients with silicosis in Hong Kong. Am Rev Respir Dis 1992; 145: 36-41.

15.- WHITTINGTON RM. Fatal hepatotoxicity of antitubercular chemotherapy. Lancet 1991; 338: 1083-4.

16.- TOST JR, VIDAL R, CAYLA J, DÍAZ-CABANELA D, JIMÉNEZ A, BROQUETAS JM. Severe hepatotoxicity due to anti-tuberculosis drugs in Spain. Int J Tuberc Lung Dis 2005; 9: 534-40.

17.- HO CC, CHEN YC, HU FC, YU CJ, YANG PC, LUH KT. Safety of fluoroquinolone use in patients with hepatotoxicity induced by anti- tuberculosis regimens. Clin Infect Dis 2009; 48: 1526-33.

18.- ORMAN ES, CONJEEVARAM HS, VUPPALANCHI R, FRESTON JW, ROCHON J, KLEINER DE, et al. Clinical and histopathologic features of fluoroquinolone-induced liver injury. Clin Gastroenterol Hepatol 2011; 9: 517-23. 
19.- LARREY D. Epidemiology and individual susceptibility to adverse drug reactions affecting the liver. Semin Liver Dis 2002; 22: 145-55.

20.- KOPANOFF DE, SNIDER DE JR, CARAS GJ. Isoniazid-related hepatitis. Am Rev Respir Dis 1978; 117 : 991-1001.

21.- VAN DEN BRANDE P, VAN STEENBERGEN W, VERVOORT G, DEMEDTS M. Aging and hepatotoxicity of isoniazid and rifampin in pulmonary tuberculosis. Am J Respir Crit Care Med 1995; 152: 1705-8.

22.- VAN HEST, BAARS H, KIK S, VAN GERVEN P, TROMPENAARS MC, KALISVAART N, et al. Hepatotoxicity of Rifampin-Pyrazinamide and Isoniazid Preventive Therapy and Tuberculosis Treatment. Clin Infect Dis 2004; 39: 488-96.

23.- GRONHAGEN-RISKA C, HELLSTROM PE, FROSETH B. Predisposing Factors in Hepatitis Induced by Isoniazid- Rifampin Treatment of Tuberculosis. Am Rev Respir Dis 1978; 118: 461-6.

24.- WURWITZ N. Predisposing factors in adverse reactions to drugs. Br Med J 1969: 1: 536.

25.- IWAINSKY H, WINSEL K, WERNER E, EULE H. On the pharmacokinetics of rifampicin during treatment with intermittent administration, Scand J Respir Dis 1976; $57: 5$.

26.- FRANKS AL, BINKIN NJ, SNIDER DE JR, ROKAW WM, BECKER S. Isoniazid hepatitis among pregnant and postpartum Hispanic patients. Public Health Rep. 1989; 104: 151-5.

27.- GORDIN FM, COHN DL, MATTS JP, CHAISSON RE, O'BRIEN RJ. For the Terry Beirn Hepatotoxicity of Rifampin and Pyrazinamide in the Treatment of Latent Tuberculosis Infection in HIV-Infected Persons: Is It Different Than in HIV-Uninfected Persons? Clin Infect Dis 2004; 39: 561-5.

28.- OHNO M, YAMAGUCHI I, YAMAMOTO I. Slow $\mathrm{N}$-acetyltransferase 2 genotype affects the incidence of isoniazid and rifampicin-induced hepatotoxicity Int $\mathbf{J}$ Tuberc Lung Dis 4: 256-61.

29.- PANDE JN, SINGH SPN, KHILNANI GC, KHILNANI S, TANDON RK. Risk Factors for Hepatotoxicity from Antituberculosis Drugs: a case-control study Thorax 1996; 51: 132-6.

30.- HUANG YS, CHEM HD, SU WJ WU JC, LAI SL, YANG SY, et al. Polymorphism of the N-acetyltransferase 2 gene as a susceptibility risk factor for antituberculosis drug-induced hepatitis. Hepatology 2002; 35: 883-9.

31.- PARKIN DP, VANDENPLAS S, BOTHA FJH, VANDENPLAS ML, SEIFART HI, VAN HELDEN PD, et al. Trimodality of isoniazid elimination. Phenotype and genotype in patients with tuberculosis. Am J Respir Crit Care Med 1997; 155: 1717-22.

32.- MITCHELL JR, ZIMMERMAN HJ, ISHAK KG, THORGEIRSSON UP, TIMBRELL JA, SNODGRASS $\mathrm{WR}$, et al. Isoniazid liver injury: clinical spectrum, pathology and probable pathogenesis. Ann Intern Med 1976; 84: 181-92.

33.- TIMBRELL JA, MITCHELL JR, SNODGRASS WR, NELSON SD. Isoniazid hepatotoxicity: The relationship between covalent binding and metabolism in vivo. $\mathrm{J}$ Pharmacol Exp Ther 1980; 213: 364-9.

34.- PERETTI E, KARLAGANIS G, LAUTERBURG BH. Acetylation of acetylhydrazine, the toxic metabolite of isoniazid, in humans. Inhibition by concomitant administration of isoniazid. J Pharmacol Exp Ther 1987; 243: 686-9.

35.- LAUTERBURG BH, SMITH CV, TODD EL, MITCHELL JR. Pharmacokinetcs of the toxic hydrazino metabolites formed from isoniazid in humans. J Pharmacol Exp Ther 1985; 235: 566-70.

36.- HUANG YS. Genetic polymorphisms of drug-metabolizing enzymes and the susceptibility to antituberculosis drug- induced liver injury. Expert Opin Drug Metab Toxicol 2007; 3: 1-8.

37.- LÖNNROTH K, WILLIAMS B, STADLIN S, JARAMILLO E, DYE C. Alcohol use as a risk factor for tuberculosis-a systematic review. BMC Public Health 2008; 8: 289.

38.- WORLD HEALTH ORGANIZATION. Alcohol training in general practice. EUR/ICP/ADA 038 (B). Copenhagen, Denmark: WHO, 1994.

39.- KOPANOFF DE, SNIDER D, CARAS G. Isoniazid related hepatitis: a U.S. Public Health Service cooperative surveillance study. Am Rev Respir Dis 1979; 117 : 991-1001.

40.- HWANG SJ, WU JC, LEE CN, YEN FS, LU CL, LIN $\mathrm{TP}$, et al. A prospective clinical study of isoniazidrifampicin-pyrazinamide- induced liver injury in an area endemic for hepatitis B. J Gastroenterol Hepatol 1997; 12: 87-91.

41.- COHN DL, CATLIN BJ, PETERSON KL, JUDSON FN, SBARBARO JA. A 62-dose, 6-month therapy for pulmonary and extrapulmonary tuberculosis: a twiceweekly, directly observed, and cost-effective regimen. Ann Intern Med 1990; 112: 407-15.

42.- SINGAPORE TUBERCULOSIS SERVICE/BRITISH MEDICAL RESEARCH COUNCIL. Clinical trial of three 6-month regimens of chemotherapy given intermittently in the continuation phase in the treatment of pulmonary tuberculosis. Am Rev Respir Dis 1985; 132 : 374-8.

43.- MAHMOOD K, HUSSAIN A, JAIRAMANI KL, TALIB A, ABBASI B, SALKEEN S. Hepatotoxicity with antituberculosis drugs: the risk factors. Pak J Med Sci 2007; $23: 33-8$.

44.- MORGAN FREIMAN J, JACOBSON KR, MUYINDIKE WR, ROBERT HORSBURGH C, ELLNER JJ, HAHN JA, et al. Isoniazid Preventive Therapy for People with HIV who are Heavy Alcohol Drinkers in High TB/HIV Burden Countries: A Risk-Benefit Analysis. J Acquir Immune Defic Syndr. 2018; 77: 405-12. 
45.- RAJANI SHAKYA, B SUBBA RAO, BHAWANA SHRESTHA. Incidence of Hepatotoxicity Due to Antitubercular Medicines and Assessment of Risk Factors Annals of Pharmacotherapy s 2004; 38: 1074-9.

46.- MEHTA S. Malnutrition and drugs: clinical implications. Dev Pharmacol Ther 1990; 15: 159-65.

47.- WONG WM, WU PC, YUEN MF, CHENG CC, YEW WW, WONG PC, et al. Antituberculosis drug-related liver dysfunction in chronic hepatitis B infection. Hepatology 2000; 31: 201-6.

48.- HOFFMANN CJ, CHARALAMBOUS S, THIO CL, MARTIN DJ, PEMBA L, FIELDING KL, et al. Hepatotoxicity in an African antiretroviral therapy cohort: the effect of tuberculosis and hepatitis B. AIDS 2007; 21: 1301-8.

49.- UNGO JR, JONES D, ASHKIN D, HOLLENDER ES, BERNSTEIN D, ALBANESE AP, et al. Antituberculosis drug-induced hepatotoxicity. The role of hepatitis $\mathrm{C}$ virus and the human immunodeficiency virus. Am J Respir Crit Care Med 1998; 157: 1871-6.

50.- JAIN MK. Drug-induced liver injury associated with HIV medications. Clin Liver Dis. 2007; 11: 615-9.

51.- NÚÑEZ M. Hepatotoxicity of antiretrovirals: incidence, mechanisms and management. J Hepatol 2006; 44: S132-S139.

52.- YILDIZ A, SEVER MS, TURKMEN A. Tuberculosis after renal transplantation: experience of one Turkish centre. Nephrol Dial Transplant 1998; 13: 1872-5.

53.- ANTONY SJ, YNARES C, DUMMER JS. Isoniazid hepatotoxicity in renal transplant recipients. Clin Transplant 1997; 11: 34-7.

54.- WRIGHT JM, STOKES EF, SWEENEY VP. Isoniazidinduced carbamazepine toxicity and vice versa: a double drug interaction. N Engl J Med 1982; 307: 1325-7.

55.- D VALIQUETTE C, PELLETIER M, PARISIEN I, ROCHER I, MENZIES D. Incidence of serious side effects from first-line antituberculosis drugs among patients treated for active tuberculosis. Am J Respir Crit Care Med 2003; 167: 1472-7.

56.- GROSSET J, TRUFFOT-PERNOT C, LACROIX C, JI
B. Antagonism between isoniazid and the combination pyrazinamide-rifampin against tuberculosis infection in mice. Antimicrob Agents Chemother. 1992; 36: 548-51.

57.- JASMER RM, SAUKKONEN JJ, BLUMBERG HM, DALEY CL, BERNARDO J, VITTINGHOFF E, et al. Short-course rifampin and pyrazinamide compared with isoniazid for latent tuberculosis infection: a multicenter clinical trial. Ann Intern Med 2002; 137: 640-7.

58.- AMERICAN THORACIC SOCIETY, CENTERS FOR DISEASE CONTROL, INFECTIOUS DISEASES SOCIETY OF AMERICA. Treatment of tuberculosis. Am J Respir Crit Care Med 2003; 167: 603-62.

59.- JOINT TUBERCULOSIS COMMITTEE OF THE BRITISH THORACIC SOCIETY. Chemotherapy and management of tuberculosis in the United Kingdom: recommendations 1998. Thorax 1998; 53: 536-48.

60.- MCNEILL L, ALLEN M, ESTRADA C, COOK P. Pyrazinamide and rifampin vs isoniazid for the treatment of latent tuberculosis: improved completion rates but more hepatotoxicity. Chest 2003; 123: 102-6.

61.- SINGANAYAGAM A, SRIDHAR S, DHARIWAL J, ABDEL-AZIZ D, MUNRO K, CONNELL DW, et al. A comparison between two strategies for monitoring hepatic function during anti-tuberculous therapy. Am J Respir Crit Care Med 2012; 185: 653-9.

62.- WATKINS PB. Biomarkers for the diagnosis and management of drug-induced liver injury. Semin Liver Dis 2009; 29: 393-9.

63.- SCHARER L, SMITH JP. Serum transaminase elevations and other hepatic abnormalities in patients receiving isoniazid. Ann Intern Med 1969; 71: 1113-20.

64.- SENIOR JR. Monitoring for hepatotoxicity: what is the predictive value of liver "function" tests? Clin Pharmacol Ther 2009; 85: 331-4.

65.- SHARMA SK, SINGLA R, SARDA P, MOHAN A, MAKHARIA G, JAYASWAL A, et al. Safety of 3 different reintroduction regimens of antituberculosis drugs after development of antituberculosis treatment-induced hepatotoxicity. Clin Infect Dis 2010; 50: 833-9.
Correspondencia a:

Dr. Fernando Tirapegui S.

Departamento de Enfermedades Respiratorias,

Pontificia Universidad Católica de Chile.

Diagonal Paraguay 362, piso 6, Santiago, Chile.

Email: ftirapegui@uc.cl 